\title{
A Tailored Cognitive Behavioural Therapy Intervention for Depression in People Newly Diagnosed with Multiple Sclerosis
}

\author{
An Expert Interview with Litza Kiropoulos \\ Melbourne School of Psychological Sciences, University of Melbourne, Victoria, Australia
}

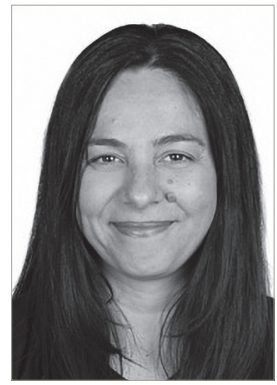

DOI: https://doi.org/10.17925/USN.2021.17.2.68

\section{Litza Kiropoulos}

Dr Litza Kiropoulos is a senior lecturer and senior clinical psychologist based in the Melbourne School of Psychological Sciences at the University of Melbourne. Dr Kiropoulos is also the director of the University of Melbourne Psychology Clinic and heads the Mood and Anxiety Disorders Lab. Dr Kiropoulos works at the nexus of research, clinical practice and clinical education. She has over 20 years of clinical experience working in public mental health. Her research interests include mood and anxiety disorders, transdiagnostic mechanisms involved in the development and maintenance of mood, anxiety and eating disorders, and the development and evaluation of psychological interventions for depression and anxiety disorders in various populations including clinical and medically ill populations including multiple sclerosis. Relating to multiple sclerosis, her research focuses on four areas: 1) optimizing psychological interventions for depression and anxiety and related issues in people with multiple sclerosis; 2) examining biomarkers of depression in people with multiple sclerosis; 3) psychological mechanisms involved in the development and maintenance of depression and anxiety and related factors (cognitive impairment, fatigue, pain, sleep disturbances) in people with multiple sclerosis; and 4) cognitive changes in people with multiple sclerosis.

\section{Keywords}

Depression, anxiety, CBT, early intervention, biomarkers, psychological mechanisms, multiple sclerosis

Disclosures: The phase II trial received funding in the form of a project grant from MS Research Australia.

Acknowledgements: Medical writing assistance was provided by Katrina Mountfort, freelancer, and funded by Touch Independent Medical Education.

Review process: This is an expert interview and, as such, has not undergone the journal's standard peer-review process.

Compliance with ethics: This article is an interview and did not involve any studies with human or animal subjects performed by the author.

Data availability: Data sharing is not applicable to this article as no datasets were generated or analysed during the current study/during the writing of this article.

Authorship: The named author meets the International Committee of Medical Journal Editors (ICMJE) criteria for authorship of this manuscript, takes responsibility for the integrity of the work as a whole, and has given final approval for the version to be published.

Received: 21 October 2021

Published online: 3 December 2021

Citation: touchREVIEWS in Neurology 2021;17(2):68-9

Corresponding author: Litza Kiropoulos,

Melbourne School of Psychological Sciences,

University of Melbourne, Victoria 3010

Australia.E: litzak@unimelb.edu.au

Support: No funding was received in

the publication of this article.
$\mathrm{M}$ ultiple sclerosis (MS) is a chronic neurological disease that is associated with high rates of depression and anxiety, ${ }^{1}$ particularly in the early stages of diagnosis. ${ }^{2,3}$ A growing body of evidence suggests that cognitive behavioural therapy (CBT) is an effective treatment for depression in people with MS. ${ }^{4,5}$ However, there is a lack of tailored CBT interventions for patients who have been newly diagnosed with MS.

In an expert interview, Litza Kiropoulos discusses the ACTION-MS study, an Australian clinical trial that is investigating the effectiveness of a tailored CBT intervention in individuals with depression and newly diagnosed MS

\section{Q. What is the prevalence of depression in people with multiple sclerosis?}

Studies have suggested that the lifetime prevalence of depression is up to $50 \%$ of the MS population (i.e. half of patients with MS will experience depression in their lifetime), but the point prevalence is up to $30 \%$, so depression has been recognized as a major issue in MS. In newly diagnosed patients, anxiety and depression increase further, up to $80 \% .^{1-3}$

\section{Q. Is depression in multiple sclerosis associated with specific changes to the central nervous system?}

Depression has also been found to be linked to neurological changes, and changes to cytokine production is also found. ${ }^{6}$

\section{Q. Is depression related to other multiple sclerosis symptoms, such as fatigue?}

Fatigue, pain and anxiety are considered risk factors for depression in MS. ${ }^{7}$

\section{Q. What is known about the effectiveness of treatment interventions for people with multiple sclerosis and depression?}

CBT-based interventions have been found to be effective in treating depression and anxiety. These include acceptance and commitment therapy, and mindfulness-based practices. My research focuses on tailored CBT interventions for patients newly diagnosed with MS. 


\section{Q. What are the aims and design of the ACTION-MS study?}

The ACTION-MS study is a phase II study involving newly diagnosed patients, and compares the tailored CBT 8-week face-to-face intervention with an active comparator arm, which is supportive listening. ${ }^{8}$ The latter is the opposite of CBT, which is structured and can be quite directive, whereas supportive listening is client led and can be quite unstructured. This study follows a phase I proof-of-concept trial. To date, 45 patients have been recruited; patients will also undergo a 3-month assessment after the intervention phase.

\section{Q. What are the outcome measures and findings of this study to date?}

The primary outcome measure is the level of depression. Findings to date show that, compared with the comparator arm, levels of depressive symptoms in patients in the CBT arm reduced by an average of at least 10 points immediately after the intervention, which was sustained at 3 months. This is a significant difference between the groups and is also clinically meaningful, as the 10 points are on the Beck Depression Inventory (BDI-II), which is considered the gold standard for measuring depression. We also saw reduced levels of anxiety following the CBT intervention.

\section{Q. What are your recommendations based on these findings?}

The feedback from patients indicates that this intervention is very much needed and should be offered as part of routine MS care, whether or not depression is an issue. Qualitative data from the trial also showed that participants were very positive about an online version of the current CBT-based psychological intervention given the current COVID-19 climate, but also if participants have issues with travelling to receive care, mobility and cost issues. The emotional aspects of receiving an MS diagnosis are not considered in current practice, and the earlier these issues are resolved, the better, because the longer depression and anxiety continues, the more difficult they are to treat.

\section{Q. When will the final results be available and what future studies are planned?}

I hope that this study will complete next year. We hope to recruit 60 patients but COVID-19 has delayed recruitment. An online version of this intervention is also planned. We hope to offer this fully automated internet-based intervention to individuals with MS. This will be assessed in a phase III clinical trial. We are also conducting the Transdiagnostic Research Program, an online study looking at transdiagnostic factors in depression, anxiety and eating disorders in individuals with MS. ${ }^{9}$

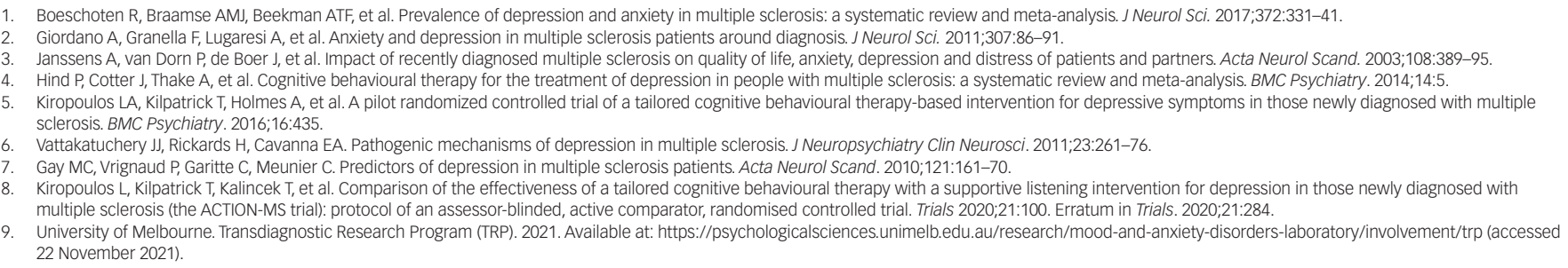

